Ann. Zootech., r973, $22(2), 219-235$.

\title{
UTILISATION DE QUANTITÉS ÉLEVÉES DE BETTERAVES PAR LES VACHES LAITIÈRES : ÉTUDE DE L'INGESTION, DE LA DIGESTION ET DES EFFETS SUR LA PRODUCTION
}

\author{
R. VÉRITÉ et M. JOURNET \\ avec la collaboration technique de Jeanne Fléchet, Renée Lefaivre, J. Lefaivre et A. Oldier \\ Station de Recherches sur l'Élevage des Ruminants \\ Centre de Recherches de Clermont Ferrand, I. N.R.A., \\ Theix, Saint Genès Champanelle, 63110 Beaumont
}

RÉSUMÉ

Au cours de 2 essais (chacun avec 2 vaches fistulées du rumen) nous avons voulu étudier la digestion (ainsi que l'ingestion et les effets sur la production) de rations contenant des betteraves en quantités croissantes (o-2,5 - 5-7,5 - Io et I $2 \mathrm{~kg}$ MS) associées à chacun des 4 fourrages suivants (tabl. I) distribués à volonté : ensilage d'herbe - foins de luzerne de qualité moyenne et de qualité excellente - ensilage de maïs. Chaque essai a duré 22 semaines.

L'étude des quantités ingérées (fig. I) a montré que la substitution des betteraves aux ensilages était totale (sur la base de la matière sèche) quelle que soit la quantité de betteraves. La substitution n'est que partielle entre les betteraves et le foin ( I kg MS de betteraves pour o,5 kg MS de foin) lorque les quantités de betteraves sont inférieures à 5 ou ro $\mathrm{kg}$ MS (selon la qualité du foin).

L'accroissement des quantités de betteraves ingérées a provoqué :

Io une diminution de l'activité cellulolytique du jus de rumen au-dessus de $5 \mathrm{~kg}$ MS de betteraves (fig. 5) et du pH du jus de rumen (fig. 6). Celui-ci est devenu inférieur à 6,5 à partir de 7 ou $8 \mathrm{~kg}$ MS de betteraves mais n'a pas atteint des valeurs très faibles (minimum : 5,9).

$2^{\circ}$ une diminution importante de la proportion relative d'acide acétique dans le rumen (jusqu'à $45 \mathrm{p}$. I 00 en moyenne pour Io $\mathrm{kg}$ MS betteraves) et une augmentation de celles d'acide butyrique ( 22 p. Ioo en moyenne) et d'acide valérianique ( 7 p. Ioo en moyenne) (fig. 8). Les différences entre vaches sont importantes. La proportion relative d'acide acétique augmente $(r=0,82)$ avec le $\mathrm{pH} d u$ jus de rumen tandis que celle d'acide butyrique diminue $(r=0,69)$. L'évolution, au cours de la journée, de la composition du mélange d'AGV du rumen est d'autant plus importante que la quantité de betteraves ingérée est plus forte (fig. 7) ;

$3^{\circ}$ une augmentation très variable de la teneur en acide lactique du jus de rumen (tabl. 2);

$4^{0}$ une augmentation parfois importante de la teneur en corps cétoniques du sang (teneurs comprises entre 2 et $I 7 \mathrm{mg} / \mathrm{IOO} \mathrm{ml}$ ) mais très variable selon Ies vaches (fig. 9). Cette teneur est surtout importante lorsque la proportion relative d'acide butyrique est supérieure à. 20 p. I.00 (fig. IO) ; 
$5^{\circ}$ une augmentation importante du taux butyreux et du taux de protéines du lait sauf avec l'ensilage de maïs (fig. 3) ainsi qu'une diminution importante de la proportion des acides gras longs $\left(\mathrm{C}_{18}\right)$ du lait et de leur quantité sécrétée (fig. 4).

Les origines et les conséquences possibles de ces différentes modifications ont été discutées ainsi que les possibilités pratiques d'utiliser des quantités élevées de betteraves dans la ration des vaches laitières.

\section{INTRODUC'TION}

En France, malgré une diminution régulière des surfaces cultivées, la betterave fourragère occupait encore en I97I une place aussi importante que le maïs fourrage ( 420 ooo ha contre 460000 ). Flle est encore souvent utilisée dans la ration des vaches laitières par suite de ses avantages agronomiques (forte production de matière sèche à l'ha) et zootechniques (forte valeur énergétique et effet favorable sur l'ingestion), de sa facilité de conservation et des progrès récents dans le domaine de sa culture. Cependant, sa richesse en saccharose en fait un aliment très particulier qui, ingéré en grande quantité, peut provoquer des ennuis sanitaires par suite de sa rapidité de fermentation (DiRksen, I970). De ce fait, elle n'est incluse qu'en quantité relativement faible dans les rations : 2 à $5 \mathrm{~kg}$ de matière sèche (MS).

Les essais de JOURnet et JARRige (non publiés) et ceux de CASTLE et al. (I96I et I963) ont permis de mettre en évidence l'effet favorable de l'addition, à la ration de fourrages, d'une quantité moyenne de betteraves (inférieure à $6 \mathrm{~kg}$ de MS) sur la quantité de matière sèche et d'énergie ingérée, la production de lait et le taux de protéines du lait. Ces avantages inciteraient à augmenter les quantités distribuées s'il n'y avait le risque d'acidose. Les quantités maximales de betteraves à ne pas dépasser et les conditions optimales d'utilisation n'ont pas été bien précisées jusqu'ici.

TABILAU I

Composition chimique des aliments

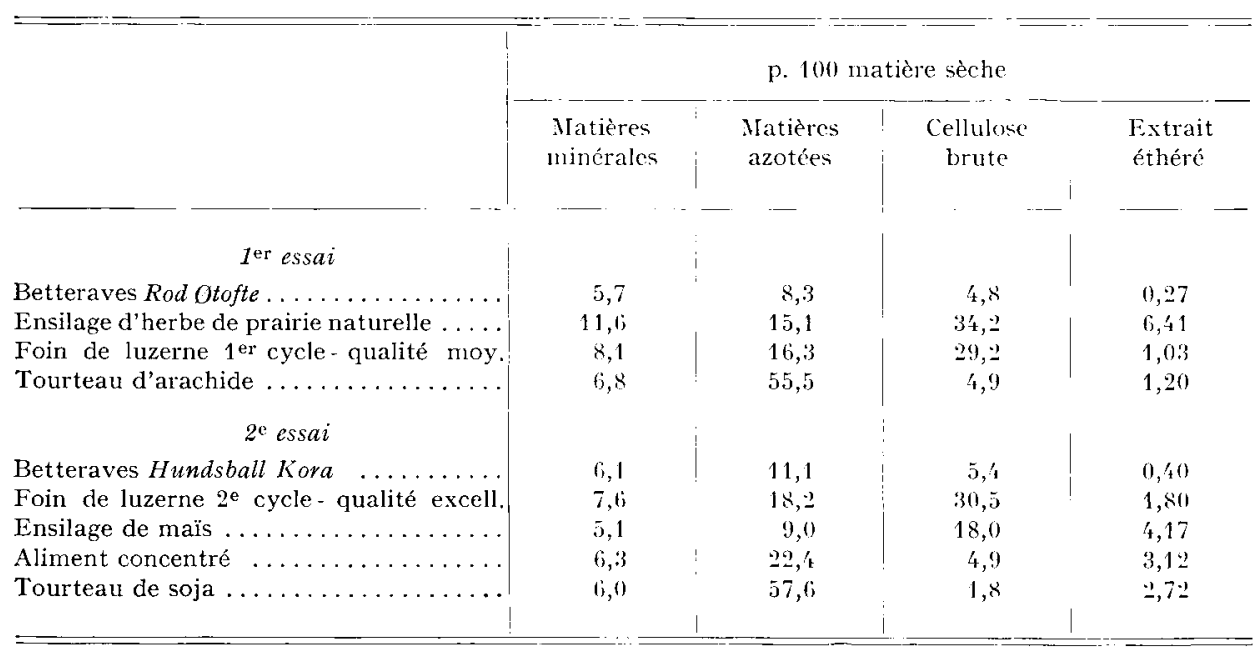


Les essais de KaUfmann et Romr (I967) et de OrTh et KaUfmann (I967) avec de fortes quantités de betteraves ont montré une diminution du $\mathrm{pH}$ du rumen et une modification importante des fermentations mais ne permettent pas de fixer une limite maximale d'utilisation. Il était donc intéressant d'étudier les effets de quantités très élevées de betteraves sur l'ingestion, la production et l'état sanitaire des animaux, en essayant d'expliquer ces effets à partir des principales modifications de la digestion et du métabolisme.

Au cours de 2 essais, avec 4 vaches fistulées en lactation, nous avons accru progressivement (en 5 ou 6 périodes) les quantités de betteraves distribuées, de o jusqu'à I2 $\mathrm{kg}$ MS. Cette étude a été faite avec chacun des 4 fourrages suivants : ensilage d'herbe, ensilage de maïs, foin de luzerne, de qualité excellente ou de qualité médiocre (tabl. I).

\section{MATÉRIEL ET MÉTHODES}

\section{Schéma expérimental, animaux, alimentation}

Au cours du I er essai (hiver 1969-1970), 2 vaches fistulées ( ${ }^{\circ}{ }^{3}$ I 65 et 4 r88), de race Frisonne Pie-Noire ( 620 et $540 \mathrm{~kg}$ ), en lactation depuis 8 et I I mois et produisant $\mathrm{I} 2 \mathrm{et} 9 \mathrm{~kg}$ de lait au début de l'essai, ont reçu un fourrage à volonté (ensilage d'herbe de prairie naturelle pour les périodes I à 6 et foin de luzerne de qualité moyenne pour les périodes 7 à $\mathrm{II}$ ) et des quantités variables de betteraves (variété Rod Øtofte, 2 I p. Ioo MS). Les quantités de betteraves distribuées ont été croissantes au cours des périodes I à $6(0-2,5-5-7,5$ - Io et I $2 \mathrm{~kg}$ MS) et décroissantes au cours des périodes 7 à II (IO- 7,5- 5- 2,5- O kg MS). Chaque période durait 2 semaines; la quantité de betteraves distribuée augmentait (ou diminuait) au cours de la première semaine et demeurait constante au cours de la $2^{\mathrm{e}}$ semaine. Lors de la $6^{\mathrm{e}}$ période, les vaches ont reçu, en plus, $\mathrm{I} \mathrm{kg}$ de tourteau d'arachide.

Au cours du $2^{\text {e }}$ essai (hiver 1970-1971), 2 autres vaches fistulées ( ${ }^{\circ} 4^{194}$ et $5^{\text {I } 23}$ ), de race Frisonne Pie-Noire (6ro et $6 \mathrm{r} 5 \mathrm{~kg}$ ), en lactation depuis 2 mois et produisant environ $20 \mathrm{~kg}$ de lait au début de l'essai, ont reçu un fourrage à volonté (foin de luzerne d'excellente qualité pour les périodes I à 6 et ensilage de maïs pour les périodes 7 à $\mathrm{r}$ I) et des quantités variables de betteraves (variété Hundsball Kora, 2 I p. Ioo MS). Les quantités de betteraves distribuées ont été croissantes $(0,7 \mathrm{~kg}$ de matière fraîche en plus chaque jour, en moyenne) au cours des périodes a à 6 (o- 2,55- 7,5- Io et I $2 \mathrm{~kg}$ MS betteraves) et des périodes 7 à I I (0- 2,5-5- 7,5- IO $\mathrm{kg}$ MS). Entre les périodes 6 et 7 une transition de 2 semaines a été ménagée. Pendant les périodes I et 2 les vaches ont reçu 5 et $2,5 \mathrm{~kg}$ d'aliment concentré pour couvrir leurs besoins et pendant les périodes 7 à II, I $\mathrm{kg}$ de tourteau de soja.

Les betteraves, hachées ( $\mathrm{r}^{\mathrm{er}}$ essai) ou entières ( $2^{\mathrm{e}}$ essai), étaient distribuées en 2 repas égaux (à 8 et $\mathrm{I} 7 \mathrm{~h}$ ) ainsi que les fourrages (à 9 et $\mathrm{i} 8 \mathrm{~h}$ ). Les vaches attachées en stalles individuelles, sur tapis de caoutchouc, disposaient d'eau en permanence.

\section{Prétèvements et mesures}

Pour chaque vache, les quantités d'aliments ingérées, la production de lait et le taux butyreux (méthode Gerber) ont été mesurés chaque jour; le taux de protéines du lait (méthode au noir amido) et le poids vif ont été mesurés une fois par semaine.

Au cours de la $2^{\mathrm{e}}$ semaine de chaque période et pour chaque vache, les mesures suivantes ont été effectuées :

- comportements alimentaire et mérycique par enregistrement des mouvements de la mâchoire (RUCKEBUSH, I963) ;

- $\mathrm{pH}\left(2^{\mathrm{e}}\right.$ essai seulement), taux et proportion des acides gras volatils du jus de rumen (par chromatographie en phase gazeuse- Rigaud et JouRNET, 1970) sur des échantillons de jus prélevés à 8, II, I 4 et I 7 heures ;

- activité cellulolytique du jus de rumen par la méthode des sachets de nylon (Journer et Demarguilly, 1967): 
- teneur en acide lactique (BARKER et SUMmerson, I94I) du jus de rumen sur des échantillons prélevés toutes les demi-heures entre 8 et I 4 heures ;

- teneur du sang en corps cétoniques sur des échantillons prélevés 3 heures après le repas (Procos, ig6I) ;

- composition en acides gras des matières grasses du lait (DEcAEN et ADDA, I97o) (sauf avec le régime d'ensilage d'herbe).

\section{RÉSULTATS}

\section{Quantités ingérées et comportement alimentaire}

Sauf dans un cas (où l'essai a dû être arrêté car la vache $n^{\circ} 5^{\text {I2}} 23$ présentait des crises de tétanie), la distribution de quantités importantes de betteraves n'a pas posé de problème sanitaire ; mais il est vrai que l'augmentation des quantités distribuées a été progressive et que les betteraves n'ont jamais été distribuées à volonté. Même avec les quantités les plus élevées, les vaches n'ont que rarement fait des refus.

Les quantités totales de matière sèche (MS) moyennes ingérées (fig. I) ont varié selon le fourrage distribué : les niveaux les plus élevés ont été enregistrés avec le

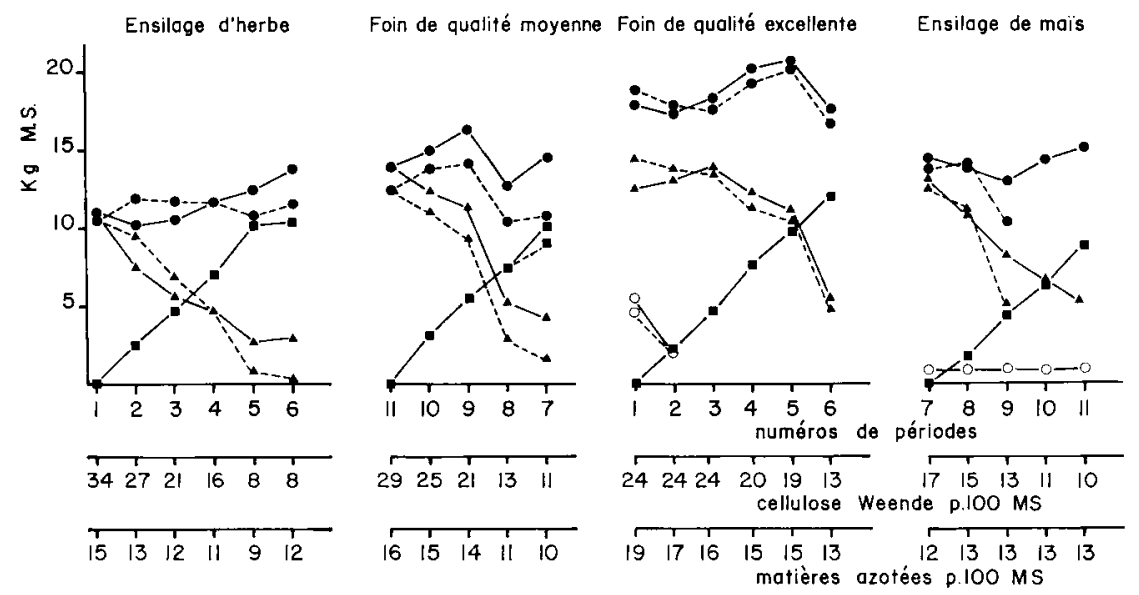

FIG. I. - Quantités de matière sèche ingérées

- Ration totale

- Fourrage grossier

- Betterave

O Aliment concentré

$$
\begin{aligned}
{ }^{e} \text { er essai }\left\{\begin{array}{l}
\text { vache } n^{\circ} 3 \text { I } 65-\ldots \\
\text { vache } n^{\circ} 4 \text { I } 88 \ldots
\end{array}\right. \\
\text { 2 }^{\text {e }} \text { essai }\left\{\begin{array}{l}
\text { vache } n^{\circ} 4 \text { I } 34-\ldots \\
\text { vache } n^{\circ} 5 \text { I } 23-\ldots
\end{array}\right.
\end{aligned}
$$

foin de luzerne de bonne qualité (20 kg MS) et les plus faibles avec l'ensilage d'herbe (II-I2 kg MS). L'augmentation de la quantité de betterave distribuée a fait augmenter la quantité de matière sèche totale ingérée de façon faible, voire nulle dans le cas des ensilages (herbe ou maïs) mais de façon relativement importante dans le cas des foins, tant que la quantité de betteraves n'a pas dépassé 5 à Io $\mathrm{kg}$ MS, selon la qualité du foin. En effet, chaque kg de MS de betteraves en supplément s'est substitué en moyenne à 0,9 à I $\mathrm{kg}$ de MS d'ensilage et à $0,5 \mathrm{~kg}$ de MS de foin (pour des quan- 
tités de betteraves allant jusqu'à $5 \mathrm{~kg} \mathrm{MS}$, avec le foin ordinaire, et jusqu'à Io $\mathrm{kg}$ MS avec le foin d'excellente qualité). Au cours des périodes de distribution maximale de betteraves (Io ou I2 $\mathrm{kg}$ MS) selon la vache et le fourrage, la consommation de fourrage a varié de 0,3 à $5 \mathrm{~kg}$ MS et la teneur en cellulose brute de la ration a varié de 6 à I3 p. Ioo.

La quantité totale d'eau ingérée (boisson + aliments) par $\mathrm{kg}$ de MS ingérée a été très constante pour chaque vache (entre 4 et $61 / \mathrm{kg}$ de MS selon les animaux) et indépendante de la proportion de betteraves dans la ration. Par contre, l'ingestion d'eau de la vache $n^{\circ} 5$ I23 a augmenté brusquement de 5,51 à 8 puis Io $1 / \mathrm{kg}$ de MS au moment de ses crises de tétanie bien que la quantité totale de MS ingérée ait alors peu diminué.

L'augmentation (fig. 2) des quantités de betteraves distribuées, de o à Io $\mathrm{kg}$ MS, avec les rations d'ensilage d'herbe, d'ensilage de maïs et de foin de qualité moyenne a provoqué une diminution des durées d'ingestion (de 7 h 55 à 5 h 45 en moyenne) et de rumination (de 9 h 30 à 7 h 23 en moyenne) et une diminution de la durée de mastication (consommation + rumination) par $\mathrm{kg}$ de MS ingérée (de $8 \mathrm{I} \mathrm{mn} / \mathrm{kg} \mathrm{MS}$ à 66 en moyenne). Ces durées sont liées à la quantité de MS de fourrage ingérée (respectivement $r=0,8 \mathrm{I} ; 0,70$ et 0,$65 ; n=26 ; \mathrm{P}<0,00 \mathrm{I}$ ) et à la teneur en
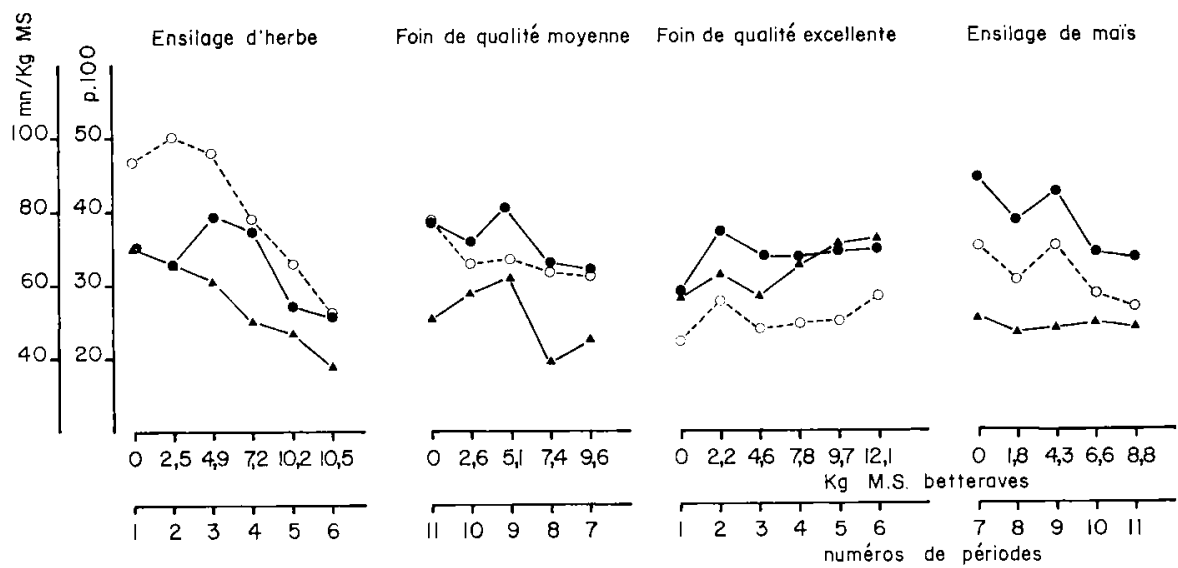

FIG. 2. - Comportement alimentaire (moyennes par régimes)

- Durée de consommation (p. Ioo des $24 \mathrm{~h}$ )

- Durée de Rumination (p. xoo des $24 \mathrm{~h}$ )

- Durée de mastication par kg de MS ingérée ( $\mathrm{mn} / \mathrm{kg}$ MS ingérée)

cellulose de la ration (respectivement $r=0,85 ; 0,67$ et 0,$70 ; n=26 ; \mathrm{P}<0,0$ ) mais pas à la quantité de matière sèche totale ingérée. Avec le foin d'excellente qualité le comportement alimentaire n'a pas été modifié par l'accroissement des quantités de betteraves distribuées. Les vaches ont ingéré entre Io et $16 \mathrm{~kg}$ de matière fraîche de betteraves entières au cours de la demi-heure suivant la distribution. La durée d'ingestion de I $\mathrm{kg}$ de MS de betteraves entières (Io à I5 mn) est donc plus grande que celle de I $\mathrm{kg}$ de MS d'aliment concentré (3 à $7 \mathrm{mn}$ selon FreER et CAMpling, I965). 


\section{Production et composition du lait}

L'expérience n'a pas été conçue pour connaître les effets des betteraves sur la production laitière ; elle met cependant nettement en évidence une diminution rapide de la production (fig. 3) au fur et à mesure de l'accroissement de la quantité de betteraves distribuée, malgré la suralimentation énergétique qui en résulte. Cette diminution peut être due en partie au type d'animal (vache fistulée), aux conditions de vie relativement peu confortables et aussi à la sous-alimentation azotée (dans le rer essai seulement).

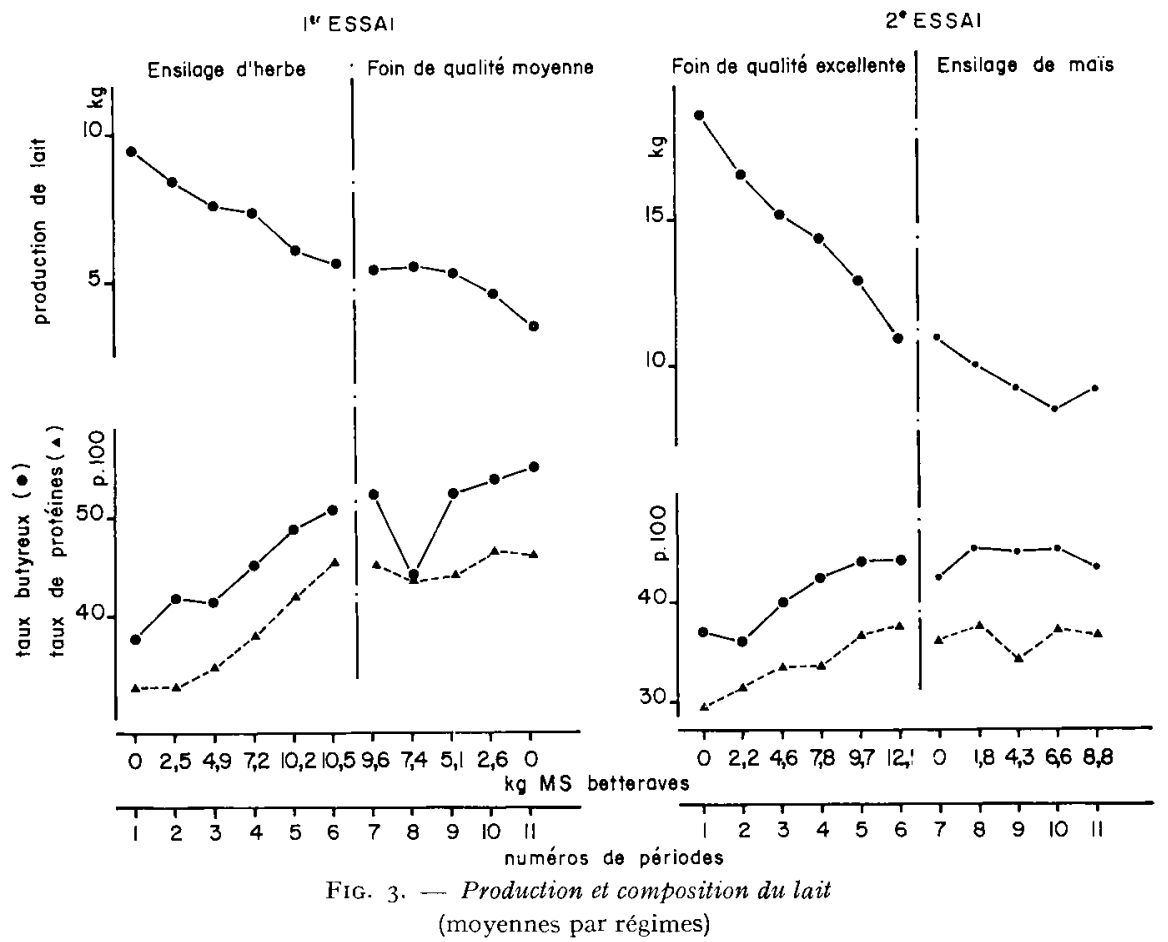

Avec l'ensilage d'herbe et le foin d'excellente qualité, l'accroissement des quantités de betteraves améliore nettement le taux butyreux et le taux de protéines du lait (fig. 4) alors que ceux-ci sont restés constants avec l'ensilage de maïs. Avec le foin ordinaire, le taux butyreux et le taux de protéines sont restés constants sans doute sous l'action combinée de deux phénomènes antagonistes : 1'effet favorable du stade avancé de lactation et l'effet défavorable de la diminution de la quantité de betterave.

Quel que soit le fourrage distribué, la composition de matières grasses du lait a été très largement dépendante de la quantité de betteraves distribuée (fig. 4). En moyenne, la proportion relative d'acides gras courts $\left(\mathrm{C}_{4}\right.$ à $\left.\mathrm{C}_{14}\right)$ augmente de même que celle d'acide palmitique. Par contre, la proportion d'acides gras longs (à I 8 atomes de carbone) diminue considérablement : elle passe de 34 à I6 p. Ioo. Dans le même temps, le rapport de l'acide oléique à l'acide stéarique $\left(\mathrm{C}_{18: 1} / \mathrm{C}_{18: 0}\right)$ a tendance 
à augmenter (de 2,5 à $4, \mathrm{I}$ ). Pour chaque groupe d'acides gras, les concentrations dans le lait et les quantités produites par jour varient de la même façon que les proportions relatives (fig. 4). Les variations sont plus importantes avec les foins qu'avec l'ensilage de maïs.

Les variations de poids vif ont parfois été très importantes d'une période à l'autre mais sont difficiles à interpréter à cause des variations de contenu digestif.
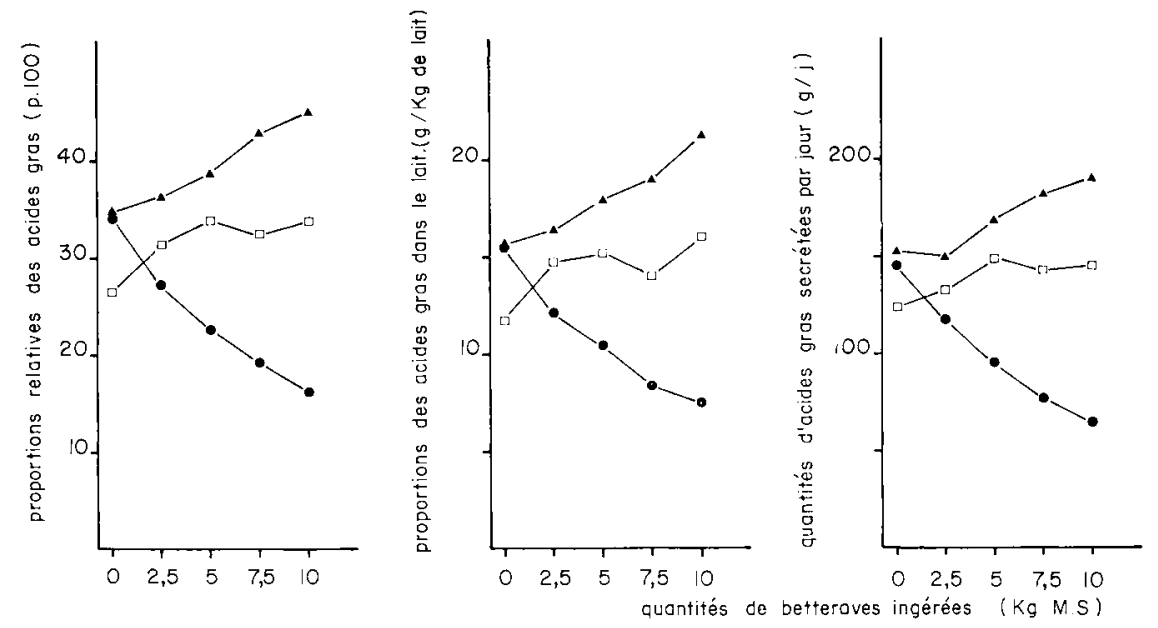

FIG. 4. - Évolution moyenne do la sécrétion des acides gras du lait

avec la quantité de betterave ingếrée

$\checkmark$ Acides comprenant 4 à I 4 atomes de carbone par molécule

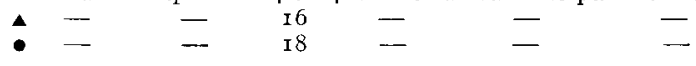

\section{Phénomènes digestifs et métaboliques}

Activité cellulolytique.

L'activité cellulolytique (fig. 5) du jus de rumen (mesurée par la proportion de papier-filtre disparue dans le rumen en $48 \mathrm{~h}$ ), variable selon les animaux, a diminué avec la teneur en cellulose brute de la ration $(r=0,85 ; n=42)$. En présence d'ensilage de maïs elle a toujours été relativement faible (20 à $50 \mathrm{p}$. Ioo de disparition). Enn présence d'ensilage d'herbe ou de foin, avec de fortes proportions de betteraves (60 à 90 p. Ioo de MS) elle a été également faible (30 à 55 p. Ioo de disparition) mais moins qu'avec des rations à forte proportion de céréales contenant 65 à 85 p.Ioo d'orge (5 à 30 p. IOo de disparition, RÉMOND et JOURNET, I972).

$p H d u$ jus de rumen.

L'évolution du pH (fig. 6) au cours de la journée a toujours été la même : maximum avant la distribution de betterave $(6,8$ à 7,6 selon la ration), le pH a été minimum $(5,9$ à 7,0$) 3$ à 6 heures plus tard avec une remontée 9 heures après la distribution.

L'accroissement de la proportion de betteraves dans la ration a provoqué une diminution du pH. Cependant, celui-ci n'a jamais été inférieur à 6,5 jusqu'à une proportion de MS de betteraves dans la ration de 45 p. IOo (7 à Io $\mathrm{kg}$ MS de bette- 
raves selon les régimes). Avec des proportions plus élevées de betteraves, le $\mathrm{pH}$ a eu un minimum plus faible $(5,9$ à 6,7$)$ et des variations plus importantes au cours de la journée (I,O à I.,2 point au lieu de 0,4 à 0,6$)$ et n'était pas encore revenu à son niveau initial 9 heures après la distribution de betteraves.

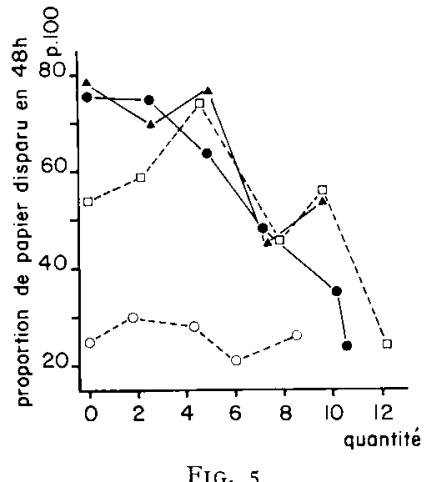

Fig. 5

FIG. 5. - Activité cellulolytique dans le rumen

- Ensilage d'herbe

- Foin de qualité moyenne

Foin de qualité excellente

o Ensilage de maís

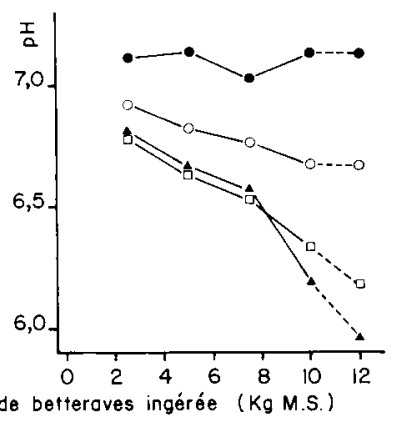

FIG. 6

FIG. 6. - $-p H$ du jus de rumen

- $8 \mathrm{~h}$

AI h

$\square \mathrm{r} 4 \mathrm{~h}$

o $17 \mathrm{~h}$

\section{Concentration et composition du mélange d'acides gras volatils du jus de rumen.}

La teneur du jus de rumen en acides gras volatils a varié au cours de la journée et a été maximale 3 à 6 heures après le repas (106 mmoles/1 en moyenne contre 76 mmoles/1 avant le repas (fig. 7). Cette teneur n'a pas été liée de façon très nette à la quantité de betteraves ingérée ; cependant, avec de fortes quantités de betteraves (7,5 ou Io $\mathrm{kg}$ de MS), elle a été, en moyenne, plus faible avant la distribution des betteraves et plus élevée 3 à 6 heures après, qu'avec de faibles quantités de betteraves (o et $2,5 \mathrm{~kg}$ MS) (fig. 7). La teneur du jus de rumen en AGV 3 heures après la distribution de betteraves a été plus élevée avec le foin de bonne qualité (I 8 mmoles) et plus faible avec le foin de qualité moyenne (96 mmoles) qu'avec les ensilages (Io6 mmoles).

La composition du mélange a évolué de façon importante au cours de la journée, surtout lorsque la quantité de betteraves était élevée : la proportion d'acide acétique a été minimale 3 heures après le repas alors que les teneurs en acides propionique, butyrique et valérianique étaient maximales, respectivement $3 \mathrm{~h}, 3-6 \mathrm{~h}$ et $6-9 \mathrm{~h}$ après le début du repas. Les modifications étant presque toutes maximales dès $3 h$, nous limiterons l'étude de l'effet des quantités de betteraves sur la composition du mélange d'AGV à ce seul prélèvement (fig. 8).

Ies différences de composition du mélange d'acides gras volatils du rumen ont été importantes entre vaches. Cependant, en moyenne, la proportion d'acide acétique diminue lorsque la quantité de betteraves augmente. Elle passe de 65-72 p. Ioo (selon les fourrages) en l'absence de betteraves, à 40-48 p. Ioo avec Io ou I $2 \mathrm{~kg}$ MS de betteraves. La proportion d'acide propionique est relativement constante; cependant, en présence d'ensilage d'herbe et de foin ordinaire, elle a nettement augmenté (maximum de 39 ou 3I p. Ioo selon la vache) quand la quantité de betteraves était 

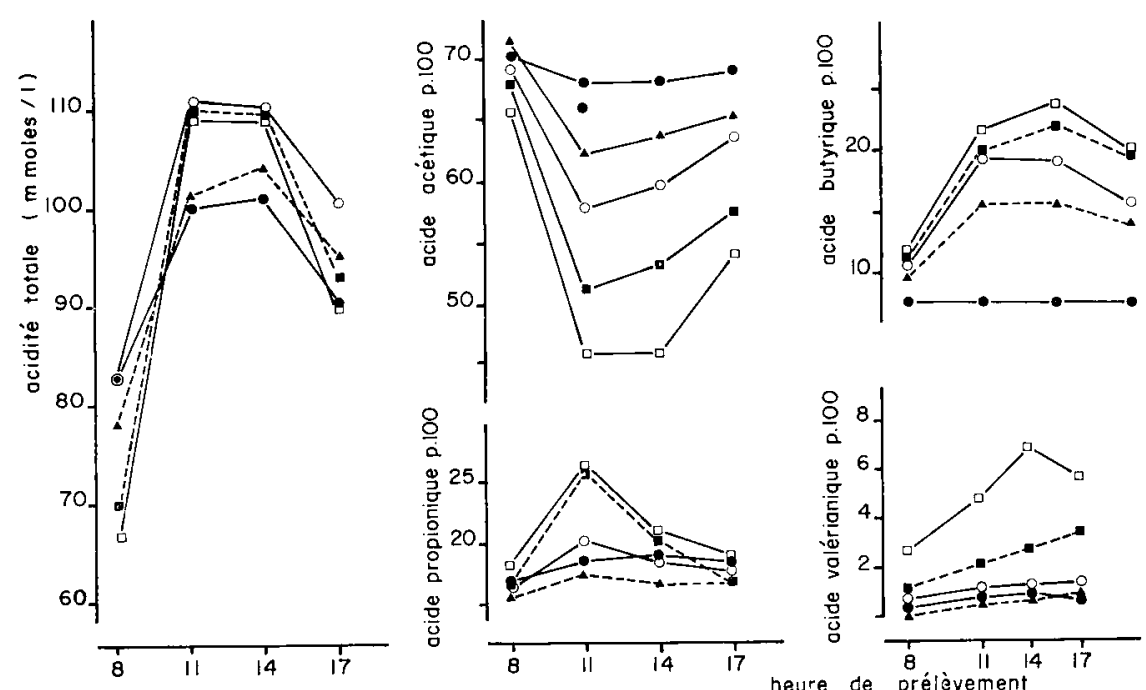

FrG. 7. - Evolution de la composition du mêlange d'acides gras volatils du rumen

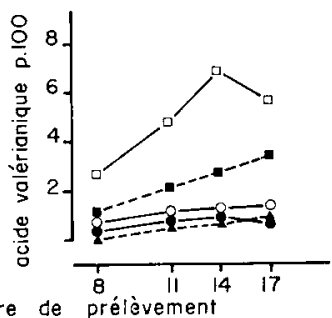
au conrs de la journée

Quantités de betteraves ingérées (kg MS)

$$
\begin{array}{ll}
\bullet=o & \square=7,5 \\
\dot{0}=2,5 & \square=\text { I0 } \\
0=5 &
\end{array}
$$

Ensiloge d'herbe
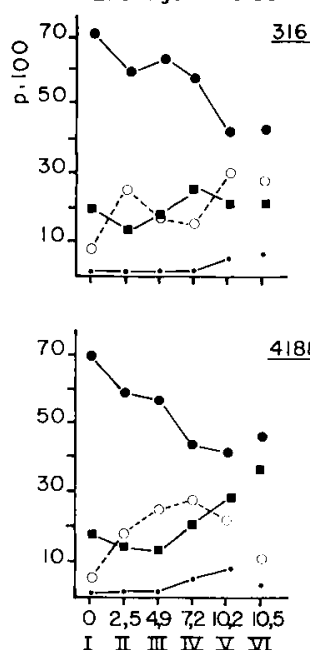

Foin de qualité moyenne Foin de qualité excellente Ensitage de mais
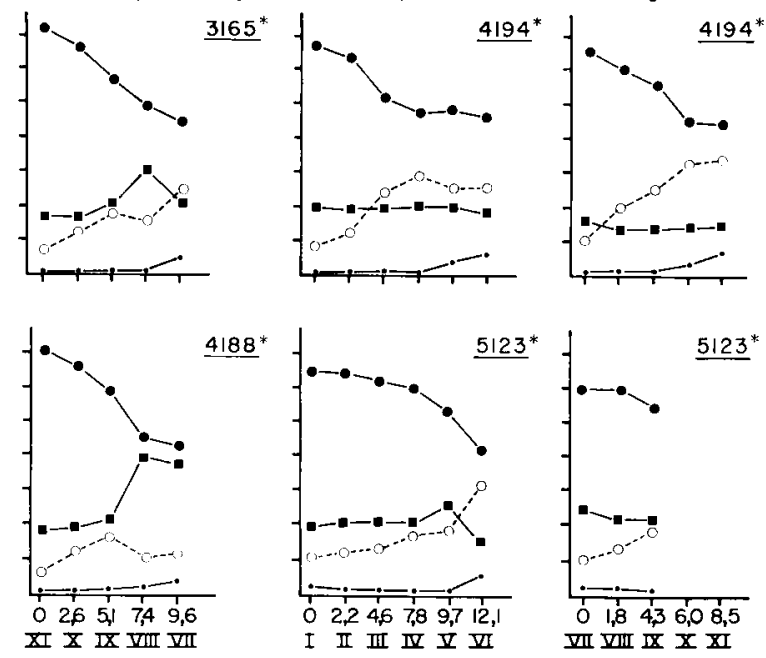

Quontités de betteraves ingérées (kg M.S.): chiffres arabes Numéros de périodes: chiffres romains

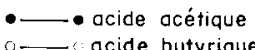

- - acide propionique * numéro de la vache

FIG. 8. - Composition en acides gras volatils du jus de vumen ( 3 heures après la distribution des betteraves) 
supérieure à $7 \mathrm{~kg}$ MS. La proportion d'acide butyrique, assez faible avec les rations sans betteraves ( 5 à Io $\mathrm{p}$. IOO), augmente en général avec la quantité de betteraves (maximum entre I 8 et $32 \mathrm{p}$. IOO). L'acide valérianique existe en quantité très importante (jusqu'à I2 p. IOO) à partir de $7 \mathrm{~kg}$ MS de betteraves, surtout en présence d'ensilage d'herbe et de foin de qualité moyenne. L'augmentation des quantités de betteraves fait diminuer la proportion d'acide isobutyrique et surtout celle d'acide isovalérianique qui passe de 2,0 à 0,4 p. Ioo. Enfin, des traces d'acide caproïque apparaissent parfois avec de fortes quantités de betteraves, notamment en présence d'ensilage de mais (entre I et 2 p. Ioo). Le rapport entre les proportions d'acide acétique et d'acide propionique diminue avec l'augmentation des betteraves dans la ration et atteint des valeurs très faibles (2, I en moyenne pour Io $\mathrm{kg}$ de MS betterave). La proportion des acides gras volatils précurseurs des matières grasses du lait (acides acétique + butyrique) est supérieure à $75 \mathrm{p}$. Ioo jusqu'à $5 \mathrm{~kg}$ MS de betteraves en présence de foin ordinaire ou d'ensilage d'herbe, 7,5 kg MS de betteraves en présence de foin excellent et ro $\mathrm{kg}$ MS de betteraves en présence d'ensilage de maîs. Les proportions relatives d'acide acétique $\left(y_{a}\right)$ et d'acide butyrique $\left(y_{b}\right)$ sont liées de façon étroite au $\mathrm{pH}(x)$ du jus de rumen :

$$
\begin{array}{lll}
y_{a}=\mathrm{I} 7,4 x-57,7 & r=0,823 & n=72 \\
y_{b}=-\mathrm{I} 3, \mathrm{I} x+\mathrm{I} 05,8 & r=0,693 & n=72
\end{array}
$$

Par contre, nous n'avons pas trouvé de liaison entre la proportion relative d'acide propionique et le $\mathrm{pH}$.

Teneur en acide lactique du jus de rumen.

L'acide lactique (tabl. 2) n'est apparu en quantité notable dans le jus de rumen qu'à partir de $5 \mathrm{~kg}$ MS de betteraves. Au-dessus de ce seuil les teneurs maximales ont varié de 0,2 à $5, \mathrm{I} \mathrm{g} / 1$. Elles ont généralement augmenté avec les quantités de betteraves ingérées mais pas de façon systématique (cf. foin excellent + I2 kg MS).

TABI,EAU 2

Teneur maximale du jus de rumen en acide lactique (g/1)

\begin{tabular}{|c|c|c|c|c|c|c|c|c|c|}
\hline \multirow{2}{*}{$\begin{array}{c}\text { Quantités } \\
\text { de } \\
\text { betteraves } \\
\text { ingérées } \\
\text { (kg M.S.) }\end{array}$} & \multirow{2}{*}{$\begin{array}{l}\text { Fourrage } \\
\text { Vache }\end{array}$} & \multicolumn{2}{|c|}{ Ensilage d'herbe } & \multicolumn{2}{|c|}{$\begin{array}{l}\text { Foin } \\
\text { de qualité } \\
\text { moyenne }\end{array}$} & \multicolumn{2}{|c|}{$\begin{array}{c}\text { Foin } \\
\text { d'excellente } \\
\text { qualité }\end{array}$} & \multicolumn{2}{|c|}{$\begin{array}{l}\text { Ensilage } \\
\text { de maïs }\end{array}$} \\
\hline & & 3165 & 4188 & 3165 & 4188 & $419 \%$ & 5123 & $419_{4}^{\prime}$ & 5123 \\
\hline 0 & & n. d. & n. d. & 0 & 0 & 0 & 0 & 0 & 0 \\
\hline & 5 & n. d. & n. d. & 0 & 0,28 & 0 & 0 & 0,20 & 0,31 \\
\hline 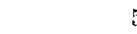 & & n. d. & n. d. & 0 & 1,08 & 0,29 & 0,31 & 0,62 & 1,33 \\
\hline & 5 & n. d. & n. d. & 0,37 & 5,12 & 0,72 & 0,18 & 0,78 & 1,74 \\
\hline 10 & & $1,86-2,06^{*}$ & $1,76-4,14^{*}$ & 3,44 & 3,96 & 2,76 & 0,62 & 1,36 & - \\
\hline 12 & & -- & - & $一$ & $\ldots$ & 0,44 & 0,44 & - & - \\
\hline
\end{tabular}

* période no 6 .

n. d. : non dosé. 
Elles ont été plus faibles lorsque la ration contenait du foin excellent ou de l'ensilage de maïs que lorsqu'elle contenait du foin ordinaire ou de l'ensilage d'herbe. Au cours de la journée, la teneur en acide lactique a été maximale 60 à $120 \mathrm{mn}$ après le début du repas de betteraves et est redevenue nulle, généralement 3 à 4 heures après le repas sauf pour une vache $\left(\mathrm{n}^{\circ} 4 \mathrm{I} 88\right)$. Les teneurs en acide lactique élevées $(>0,5 \mathrm{~g} / 1)$ ont correspondu à des $\mathrm{pH}$ faibles $(<6,6)$. Les proportions d'acide propionique et d'acide butyrique n'ont pas pu être reliées à la teneur en acide lactique du jus de rumen.

\section{Teneur en corps cétoniques du sang.}

La teneur en corps cétoniques du sang (exprimée en mg d'acétone par Ioo $\mathrm{ml}$ ), très variable $(\mathrm{I}, 7$ à $\mathrm{I} 7,4 \mathrm{mg} / \mathrm{I} 00 \mathrm{ml}$ ), a généralement (mais pas systématiquement) augmenté avec la quantité de betteraves distribuée pour atteindre parfois des valeurs très élevées (fig. 9). La teneur en acide acétoacétique n'a pratiquement pas varié $(0,4 \mathrm{mg} /$ roo $\mathrm{ml})$. L'acide $\beta$-hydroxybutyrique a représenté entre 75 et $90 \mathrm{p}$. Ioo des corps cétoniques totaux. Pour des quantités faibles ou moyennes de betteraves,
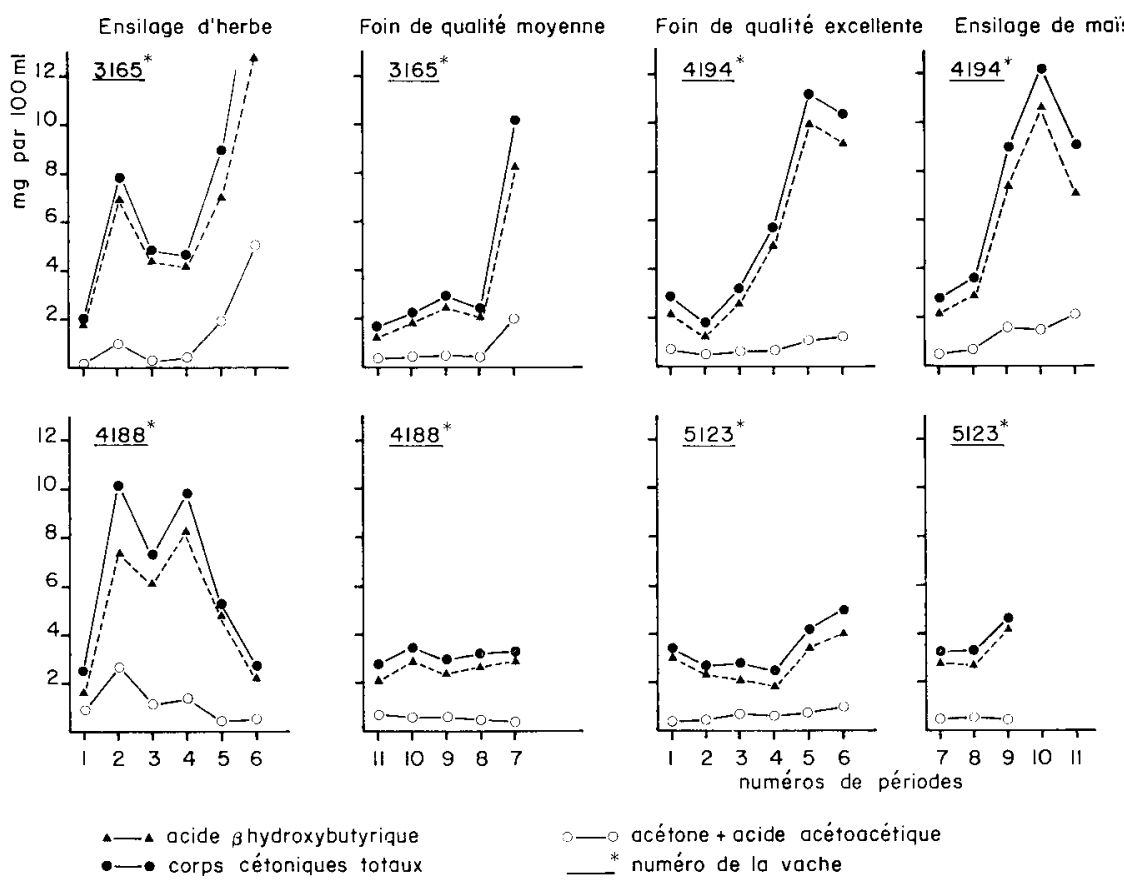

FIG. 9. - Teneur en corps cétoniques du sang

(exprimée en $\mathrm{mg}$ d'acétone par ıoo $\mathrm{ml}$ de sang)

la teneur en corps cétoniques a été nettement plus élevée avec les ensilages (surtout avec celui d'herbe) qu'avec les foins (fig. 9). Pour un même fourrage, cette teneur a varié de façon très importante entre vaches (tout comme la proportion relative d'acide butyrique dans le rumen) surtout avec les fortes quantités de betteraves. 
D'ailleurs la teneur en corps cétoniques dans le sang a été étroitement liée à la proportion relative d'acide butyrique dans le rumen (fig. Io). La teneur en corps cétoniques du sang augmente de façon lente avec la proportion relative d'acide butyrique dans le rumen tant que celle-ci ne dépasse pas $20 \mathrm{p}$. $100(r=0,55 ; n=26$;

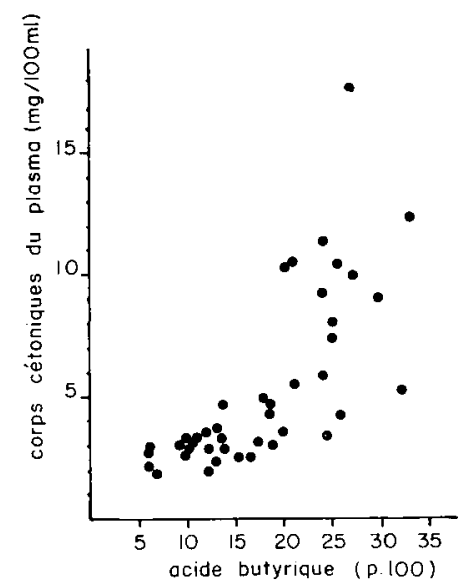

FIG. ro. - Relation entre la teneur en corps cétoniques du sang

et la proportion relative d'acide butyrique dans le rumen

$\mathrm{S}_{y x}=0,7 \mathrm{mg} / \mathrm{Io0} \mathrm{ml}$ ). Au-delà de ce point la teneur en corps cétoniques augmente de façon importante en moyenne (mais très variable) avec la proportion relative d'acide butyrique dans le rumen. Il est possible qu'à partir de ce point, la production de corps cétoniques à partir de l'acide butyrique dépasse la capacité maximale d'utilisation par l'organisme. REID et HINks (I962), sur brebis, ont observé une évoIution semblable de la teneur en corps cétoniques avec la teneur en acides gras libres du plasma. Dans ce cas, la cause première de ce phénomène serait selon BERGMAN (I97I) une déviation du métabolisme hépatique des acides gras.

\section{DISCUSSION}

Cet essai a permis de faire ingérer des quantités très élevées de betteraves (jusqu'à I2 kg MS par jour) et donc de saccharose (jusqu'à $8,5 \mathrm{~kg}$ MS par jour) à des vaches laitières fistulées en ne provoquant des troubles sanitaires qu'à une seule occasion. Cette forte ingestion de betteraves a provoqué des modifications importantes dans les fermentations du rumen et dans la sécrétion des matières grasses.

\section{Phénomènes digestifs.}

Le $\mathrm{pH}$ a diminué quand les betteraves ont augmenté dans la ration mais n'a cependant jamais atteint des valeurs très faibles (comme dans le cas d'ingestion importante de céréales), malgré la rapidité de l'ingestion et la quantité très élevée 
de glucides fermentescibles ingérée. KaUFMans et RoHR (r967) avec des betteraves, et Eirias et al. (I968) avec de la mélasse n'ont pas enregistré de $\mathrm{pH}$ faibles, non plus. WHITELAW et al. (1970) pensent que le $\mathrm{pH}$ est plus élevé en présence de protozoaires dans le rumen qu'en leur absence car ils peuvent stocker rapidement une partie des glucides fermentescibles et les soustraire ainsi à l'attaque immédiate des bactéries. Dans nos essais, 1'accroissement très progressif des quantités de betteraves offertes aux animaux a sans doute permis le maintien des protozoaires comme dans l'essai de EAdie et al. (1970) avec de l'orge. Par ailleurs, Fauconneau (I96I) et Jouany (non publié) signalent, avec des rations analogues aux nôtres, une prolifération importante des protozoaires et JouANy (non publié) observe des teneurs faibles en saccharose, glucose et fructose libres dans le jus de rumen.

Les régimes à forte proportion de glucides fermentescibles du type amidon (rations à forte proportion de céréales) ou saccharose (betteraves : KAUFMANN et RoHR, I967 ; mélasse : MARTY et PRESTON, I970 ; rations enrichies en saccharose : ØRSKOV et OLTJEN, I967 et BROSTER et al., I970) conduisent à une proportion relative d'acide acétique dans le jus de rumen très faible (souvent comprise entre 45 et 60 p. IOO). Mais avec les régimes à base d'amidon, la fermentation est généralement de type propionique, sauf quelques exceptions (EADIE et al., I970 et RÉmonD et JOURNET, 1972) alors qu'avec les régimes à base de saccharose (en particulier dans les 2 essais présents) elle est généralement de type butyrique. Les différences de pH et de flore dans le rumen en sont probablement une des causes. Nous avons en effet observé, entre le $\mathrm{pH} 6$ et 7,5, une liaison inverse entre $\mathrm{pH}$ et proportion d'acide butyrique, ce qui confirme l'hypothèse de KAUFMANN et ROHR (I967) selon laquelle l'acide butyrique se formerait surtout entre des $\mathrm{pH}$ de 6,0 et 6,5. Par ailleurs EAdie et al. (I970) ont montré avec des régimes d'orge, que le maintien de la population de protozoaires ciliés dans le rumen semble entraîner une forte proportion d'acide butyrique et une faible valeur du rapport acide propionique/acide butyrique comme dans l'essai présent où les valeurs minimales de ce rapport ont généralement été comprises entre 0,35 et 0,70 .

La formation importante d'acide valérianique a déjà été trouvée, à un degré moindre, par Demarquilly (I972) avec de fortes quantités de betteraves et par MARTY et Preston (I970), ØrSkov et OlTJEN (I967) et Broster et al. (I970) avec de la mélasse ou du saccharose. D'après ØRskov et al. (I967), cette augmentation de l'acide valérianique pourrait provenir de la présence d'acide lactique ou d'éthanol. Cependant, nous n'avons pas pu trouver de liaison entre teneurs en acide lactique et en acide valérianique. Il est possible que les rations de betteraves conduisent à la formation d'alcool puisque la présence d'éthanol dans le rumen a été notée par Ørskov et OLTJEN (I967) avec des rations purifiées contenant du saccharose ou du glucose.

La diminution de la proportion relative des acides gras volatils ramifiés (isobutyrique et surtout isovalérianique) avec l'augmentation des betteraves dans la ration pourrait être due en partie à la diminution simultanée de la teneur en azote total et en protéines de la ration et donc des acides aminés précurseurs (KAUFMANN et RoHR, I967 et $\emptyset_{\mathrm{RSKOV}}$ et Or,TJEN, I967). Elle peut également refléter une plus grande utilisation de ces acides par les bactéries par suite de l'augmentation de l'activité fermentaire. En effet, la diminution de ces acides est systématique et importante quand on passe de o à $5 \mathrm{~kg}$ MS de betteraves, même si la teneur en azote de la ration reste constante avec l'ensilage du maïs) ou élevée (avec le foin de bonne qualité). 


\section{Production et composition du lait}

Le faible nombre de vaches et leur mode de stabulation limite la portée des résultats de production. Cependant, la diminution rapide de la production de lait concorde avec les observations faites par différents auteurs avec des quantités moyennes de saccharose (betteraves : OBRACEvic et BACVANSKI, I97I ; mélasse : LOFGREEN et OtAgaki, I96o ; RoDriguez et Preston, I969; Wing et POWELl, I969 ; saccharose : Broster et al., I970). Cette diminution peut provenir des modifications des phénomènes digestifs et métaboliques ou d'effets parapathologiques. L'augmentation du taux de protéines peut être attribuée comme dans les essais de JARRIGE et JoURNET (non publiés) et de CASTLE (I96I-I963) à l'augmentation du niveau des apports énergétiques.

Sauf avec l'ensilage de maïs, l'accroissement de la quantité de betteraves a eu un effet favorable sur le taux butyreux malgré les diminutions importantes de l'apport de matières grasses alimentaires (minimum de 45 à $\mathrm{x} 70 \mathrm{~g}$ " d'extrait éthéré " par jour selon les vaches et les fourrages) du taux de cellulose (minimum de 6 à I3 p. roo) et du rapport acide acétique/acide propionique dans le rumen. Le taux butyreux n'a pas varié dans le cas de l'ensilage de maïs, probablement parce que celui-ci permet déjà lui-même des taux butyreux élevés (VÉRITÉ et JOURNET, I97I).

L'effet favorable des betteraves sur le taux butyreux peut être dû̀ à une diminution de la dilution des matières grasses produites (puisque la production de lait diminue rapidement et (ou) à une augmentation relative de la synthèse des matières grasses. Cette dernière hypothèse est confirmée par les modifications dans les acides gras du lait entraînées par l'augmentation des quantités de betteraves. En effet, les acides gras courts $\left(\mathrm{C}_{4} \mathrm{à}_{14}\right)$ et l'acide palmitique, synthétisés entièrement ou partiellement dans la mamelle augmentent non seulement en proportion relative dans les matières grasses mais aussi en concentration dans le lait et en quantité produite chaque jour. L'accroissement de la synthèse d'acides gras dans la mamelle parallèlement à la quantité de betteraves ingérée peut résulter de l'augmentation des précurseurs disponibles (la part relative des acides acétique + butyrique dans le rumen reste élevée et la teneur du sang en corps cétoniques augmente). Il est possible également que la diminution de la disponibilité des acides gras longs du sang (par suite de la diminution des apports ainsi que de la mobilisation des lipides corporels) provoque une augmentation compensatrice de l'incorporation des acides gras courts dans les triglycérides du lait (MOORE et STEELE, Ig68), ce qu'on n'observe pas avec les rations de céréales. Enfin, d'après les essais de Ørskov et al. (I967), de PradhaN et HEMKEN (I970) et de HøJ AND FREDERIKSEN et OCHIA (I970) la présence d'éthanol augmenterait le taux butyreux et provoquerait une diminution de la proportion relative de l'acide oléique comme dans l'essai présent.

\section{CONCLUSION}

L'utilisation de fortes quantités de betteraves (supérieures à 5-6 $\mathrm{kg}$ de MS) dans la ration des vaches laitières est probablement possible, à condition de réaliser une adaptation très progressive et de les associer à un foin de bonne qualité, distri- 
bué à volonté ; les ensilages (surtout celui d'herbe) ne sont pas conseillés en raison des risques de production excessive de corps cétoniques. Cependant la grande variabilité des réponses (du point de vue des phénomènes digestifs) entre vaches peut être un facteur limitant à l'emploi de quantités élevées de betteraves. A l'inverse des rations à forte proportion de céréales, celles à forte proportion de betteraves permettent des taux butyreux normaux ou même élevés, mais la production laitière semble diminuer rapidement. Des essais sur animaux en début ou milieu de lactation seront nécessaires pour confirmer ces résultats.

Reçu pour publication en décembre 1972.

\title{
SUMMARY
}

\author{
UTILIZATION OF HIGH AMOUNTS OF BEETS BY DAIRY COWS : \\ STUDY OF THE VOLUNTARY INTAKE, \\ DIGESTION AND EFFECTS ON PRODUCTION
}

\begin{abstract}
During the course of two trials (with two rumen fistulated cows per trial) we studied digestion (as well as voluntary intake, effects on production and milk composition) of a diet containing increasing amounts of beets $(0-2.5,7.5$, Io and I $2 \mathrm{~kg} \mathrm{DM})$ associated with each of the 4 following forages (table $\mathbf{r}$ ) offered ad libitum : grass silage - lucerne hay of medium or excellent quality - maize silage. Each trial lasted 22 weeks.

Study of the feed intake (fig. I) revealed that the replacement of silages by beets was total (as related to dry matter) whatever the quantity of beets used. The substitution was only partial between beets and hay ( $\mathrm{kg}$ DM of beets versus $0.5 \mathrm{~kg}$ DM of hay) when the amounts of beets were lower than 5 or Io $\mathrm{kg}$ DM (according to hay quality).
\end{abstract}

Increase of the intake of beets resulted in :

$I^{0}$ Decrease of cellulolytic activity in the rumen liquor when the intake exceeded $5 \mathrm{~kg}$ DM of beets (fig. 5) and of rumen liquor $\mathrm{pH}$ (fig. 6). The latter became lower than 6.5 from 7 or $8 \mathrm{~kg}$ DM of beets, but did not attain very low values (minimum : 5.9).

$2^{\circ}$ A large decrease in the relative proportion of acetic acid in the rumen (till $45 \mathrm{p}$. Ioo, for Io $\mathrm{kg} \mathrm{DM}$ of beets) and an increase of the proportion of butyric acid (until $25 \mathrm{p}$. Ioo) and valerianic acid (until 7 p. IOo). There were great differences between the cows. The relative proportion of acetic acid increased $(r=0.82)$ with rumen liquor $\mathrm{pH}$, whereas that of butyric acid decreased $(r=0.69)$. Variation, during the day, in the composition of the volatile fatty acid mixture was the highest when the amounts of beets eaten was large (fig. 7 ).

$3^{\circ}$ A much variable increase of the lactic acid content in the rumen liquor (table 2).

$4^{\circ} \mathrm{A}$ sometimes large increase in the content of ketones in the blood (contents between 2 and $17 \mathrm{mg} / 100 \mathrm{ml}$ ), but much variable according to cows (fig. 9). This content was particularly high when the relative proportion of butyric acid exceeded $20 \mathrm{p}$. Ioo (fig. Io).

$5^{\circ}$ A large increase of butterfat and milk protein level except in the case of maize silage (fig. 3) as well as a large decrease in the proportion of long chain fatty acids $\left(\mathrm{C}_{18}\right)$ in the milk and of the amounts secreted (fig. 4).

The origins and possible consequences of these different changes have been discussed as well as the practical use of high amounts of beets in the diets of dairy cows.

\section{RÉFÉRENCES BIBLIOGRAPHIQUES}

Barker S. B., Summerson W. M., I94I. The colorimetric determination of lactic acid in biologica material. J. Biol. Chem., 138, 535 .

Bergman E. N., I97I. Hyperketonemia-ketogenesis and ketone body metabolism. J. Dairy Sci., 54, $936-948$. 
Broster W. H., Sutton J. D., Smith T., Broster V. J., Balch C. C., Ig7o. The effect of supplements of sucrose and of glucose monohydrate on the milk production and live weight of dairy cows. J. Agric. Sci. Camb., 74, 2 I7*225.

Castle M. E., DRYSDale A. D., Waite R., I96I. The effect of root feeding on the intake and production of dairy cows. J. Dairy Res., 28, 67-74.

Castle M. E., Drysdale A. D., Waite R., Watson J. N., ig63. The effect of replacement of concentrates by roots on the intake and production of dairy cows. J. Dairy Res., 30, 199-207.

Decaen C., AdDa J., I970. Évolution de la sécrétion des acides gras des matières grasses du lait au cours de la lactation de la vache. Ann. Biol. anim. Bioch. Biophys., 10, 659-677.

Demarouilly C., I972. Digestibilité, valeur nutritive et ingestibilité des betteraves de différentes teneturs en matière sèche. Ann. Zootech., 21, 4I5-428.

Dirksen G., 1970. Acidosis, in Physiology of digestion and metabolism in the ruminant by Phillipson A. T. Proc. of the 3rd Int. Symp. Cambridge, rg69.

Eadie J. M., Hyldgaard-Jensen J., Mann S. O., Reid R. S., Whitelaw F. G., i97o. Observations on the microbiology and biochemistry of the rumen in cattle given different quantities of a pelleted barley ration. Br. J. Nutr., 24, I57-I77.

Elias A., Preston T. R., Willis M. B., Sutherland T. M., i968. By-products of sugar-cane and intensive beef production. 4 . Fattening bulls with molasses and urea in place of grain in diets with little fiber. Rev. Cubana Cienc. agric., 2, 59-67.

Fauconneau G., I96I. Les bactéries et les protozoaires libres du rumen. Variations avec le régime alimentaire et le repas. Ann. Nutr. Aliment, 15, 29I-299.

Freer M., Campling R. G., 1965. Factors affecting the voluntary intake of food by cows. 7. The behaviour and reticular motility of cows given diets of hay, dried grass, concentrates and ground, pelleted hay. Brit. J. Nutr., 19, 195-207.

Hojland Frederiksen J., Ochia B. A., r97o. The effect of ethanol and acetic acid on milk yield and milk composition of cows given rations high in concentrates. Acta. Agriculturae. Scandinavica, 20, I7-24.

Journet M., Demarouilly C., I967. Valeur alimentaire des foins condensés. II. Influence du broyage et de la mise en agglomérés sur la digestion du foin de luzerne dans le rumen. Ann. Zootech. 16, 307-321.

KAUFMANN W., Rohr K., I967. Ergebnisse gaschromatographischer bestimmung der flüchtigen fettsäuren im pansen bei unterschiedlicher futterung. Z. Tierphysiol. Tierernähr. Futtermittelk, 22, I-8.

LofGreEN G. P., OTAGAKI K. K., I960. The net energy of blackstrap molasses for lactating dairy cows. J. Dairy Sci, 43, 220-230.

Marty R. J., Preston T. R., i97o. Molar proportions of the short chain volatile fatty acids (VFA) produced in the rumen of cattle given high-molasses diets. Rev. Cubana Cienc. agric. (Engl. ed.), 4, I 83 -I 86.

Moore J. H., Steele W., ig68. Dietary fat and milk fat secretion in the cow. Proc. Nutr. Soc., 27, 66-7o.

Obracevic C., Bacvanski S., I97I. Green sugar beet (whole plant) in rations of fattening and dairy cattle in $X^{\mathrm{e}}$ Congrès International de Zootechnie. Versailles I97I.

ØRskov E. R., Flatt W. P., Moe P. W., Oltjen R. R., i967. Caproic acid and ethanol in ruminal ingesta of cattle receiving purified diets. J. Dairy Sci., 50, 239-242.

$\oint_{\text {Rskov }}$ E. R., НемкеN R. W., Moore L. A., I967. Effect of ethanol infusion on milk fat content and composition and on volatile fatty acids in rumen liquor. J. Dairy Sci., 50, 692-695.

ØRSkov E. R., OLtJEN R. R., I967. Influence of carbohydrate and nitrogen sources on the rumen volatile fatty acids and ethanol of cattle fed purified diets. J. Nutr., 93, 222-228.

ORTh A., KaUfManN W., 1967. Zur wirkung hoher rübenmengen auf speichelfuss, pansenmotorik und pansenfermentation beim tind. Das Wirtscha. Futter., 13, 28-33.

Pradhan K., HEMKeN R. W., I970. Utilization of ethanol and its effect on fatty acid patterns in ruminants. J. Dairy Sci., 53, I739-1 756 .

Procos J., I96I. Modifications of the spectrophotometric determination of ketones bodies in blood enabling the total recovering of betahydroxybutic acid. Clin. Chem., 7, 97.

ReID R. L., Hinks N. T., I962. Studies on the carbohydrate metabolism of sheep. XIX. The metabolism of glucose, free fatty acids and ketones bodies after feeding and during fasting or undernourishment of non-pregnant, pregnant and lactating ewes. Austr. J. Agric. Res., 13, II 24 -I I36.

RÉmond B., Journet M., r972. Alimentation des vaches laitières avec des rations à forte proportion d'aliments concentrés. II. Comportement alimentaire et digestion dans le rumen. Ann. Zootech., 21, I9I-205.

Rigaud J,, Journet M., r970. Méthode de dosage des acides gras volatils dans le liquide du rumen. Ann. Biol. anim. Bioch. Biophys., 10, $15 \mathrm{I}-\mathrm{1} 57$.

Rodriguez V., Preston T. R., ig69. The relative value of final molasses and ground maïze with different proportions of crude proteins and NPN for milk producion. Rev. Cubana Cienc. agric., 3, 76 (Abstr.),

Ruckebush Y., 1963. Recherches sur la régulation centrale du comportement alimentaire chez les ruminants. Thèse Doct. Sci., Lyon. 
Verité R., Journet M., I97i. Utilisation comparée de l'ensilage de maïs et de l'ensilage d'herbe pour la production laitière. Ann. Zootech., 20, 153-167.

Wing J. M., Powell G. W., I969. Response of lactating cows to two levels of mill-run blackstrap molasses from cane grown on organic soil. J. Dairy Sci., 52, I4I3-I4I4.

Whitelaw G. F., Hyldgatrd-Jensen J., Reid R. S., Kay M. G., I97o. Volatile fatty acid production in tha rumen of cattle given an all concentrate diet. Br. J. Nutr., 24, I79-I95. 